\title{
Saber teórico y utilización de la teoría del confort en la práctica de enfermería
}

Aguayo-Verdugo, Natalia'; Valenzuela-Suazo, Sandra²

\section{RESUMEN}

Introducción: Durante años Enfermería ha luchado por el reconocimiento como disciplina, en donde el conocimiento teórico propio y la práctica basada en evidencia son requisitos fundamentales. Objetivos: 1) Identificar conocimiento sobre teorías generales de Enfermería y teorías de rango medio (TRM), su utilización en la práctica y ejemplos de aplicación de la teoría del confort de Kolcaba en el quehacer de Enfermería en la Unidad de Pabellón, Anestesia y Recuperación en un hospital de Concepción; 2) Realizar una intervención educativa como formación continua de estos profesionales. Metodología: Proyecto piloto de intervención en gestión educativa, realizado entre abril y mayo del 2017, aplicación de cuestionario inicial, seguido de educación y grupo focal, terminando con cuestionario final. Resultados: Participaron 11 enfermeros/as. El conocimiento previo a la intervención fue escaso sobre grandes teorías y nulo sobre TRM. Definieron las teorías de Enfermería como base del quehacer profesional, pero sólo 4 de los 11 profesionales reconocieron utilizarlas diariamente. Luego de la intervención educativa, identificaron ejemplos de aplicación para la teoría de Kolcaba; confort físico: valoración y manejo del dolor; confort psicoespiritual: comunicación directa con el paciente, explicando procesos a enfrentar; confort sociocultural: informar a la familia; confort ambiental: disminución de la intensidad de las luces, ruidos y/o conversaciones. Conclusiones: Se recomienda realizar intervenciones para reforzar el conocimiento disciplinar de Enfermería e incentivar la formación de grupos de trabajo entre enfermeros/as clínicos y académicos para realizar investigación, buscando disminuir la brecha existente entre el conocimiento teórico y práctico.

Palabras clave: Enfermería; Educación Continua; Comodidad del Paciente; Teoría de Enfermería (DeCS; BIREME).

`Estudiante de Magíster en Enfermería. Facultad de Enfermería, Universidad de Concepción. Facultad de enfermería casilla 160-C, Concepción, Chile. Correo electrónico: nataliaguayo@udec.cl Número de registro ORCID: 0000-0001-8383-1093

²Doctora en Enfermería. Facultad de Enfermería, Universidad de Concepción. Facultad de enfermería casilla 160-C, Concepción, Chile. Correo electrónico: svalenzu@udec.cl Número de registro ORCID: 0000-0002-1308-4835

Recibido: 19/12/2018

Aceptado: 22/03/2019

*Autor para correspondencia

\section{Cómo citar este artículo}

Aguayo-Verdugo N, Valenzuela-Suazo S Saber teórico y utilización de la teoría del confort en la práctica de enfermería. SANUS. 2019;(9): 26-37. [Acceso_- $\overline{\text { mes }} \frac{}{\text { díaño }}$; Disponible en: 


\title{
Conhecimento teórico e utilização da teoria do conforto na prá- tica de enfermagem
}

\section{RESUMO}

\begin{abstract}
Introdução: Por anos, Enfermagem tem lutado para obter o reconhecimento como sendo uma disciplina em que o próprio conhecimento teórico e prática baseado em evidências

são requisitos fundamentais. Objetivo: 1) Identificar conhecimentos sobre teorias gerais e midrange de enfermagem (TRM por sua sigla em espanhol), sua utilização na prática e exemplos de aplicação da teoria do conforto de Kolcaba nas atividades de enfermagem na ala de anestesia e recuperação de um hospital em Concepción; 2) Realizar uma intervenção educativa como formação contínua desses profissionais. Metodologia: Projeto-piloto de intervenção em gestão educacional, realizado entre abril e maio de 2017, incluindo a aplicação do questionário inicial, seguido por grupo de educação e grupo de foco, terminando com um questionário final. Resultados: Participação de 11 enfermeiros. Conhecimento prévio à intervenção era escasso em relação a grandes teorias e nulo sobre o TRM. As teorias de enfermagem foram definidas como base das atividades profissionais, mas apenas quatro 4 dos 11 profissionais reconheceram usá-los diariamente. Após intervenção educativa, exemplos da aplicação da teoria de Kolcaba foram identificados; conforto físico: avaliação e manejo da dor; conforto psicoespiritual: comunicação direta com o paciente, explicando processos para passar; conforto sociocultural: informando a família; conforto ambiental: redução da intensidade das luzes, ruídos e / ou conversas. Conclusões: Recomenda-se a realização de intervenções para reforçar o conhecimento sobre a enfermagem e incentivar a formação de grupos de trabalho entre enfermeiras clínicas e acadêmicos a fim de realizar investigação, olhando para reduzir a lacuna de existência entre conhecimento teórico e prático.
\end{abstract}

Palavras chave: Enfermagem; Educação Continuada; Conforto do Paciente; Teoria de Enfermagem (DeCS;BIREME).

\section{INTRODUCCIÓN}

El profesional de Enfermería utiliza la investigación en Enfermería basada en el conocimiento propio, como evidencia para el razonamiento crítico y la toma de decisiones en el quehacer diario. Cuando utilizan la teoría y las evidencias basadas en ella para guiar su práctica mejora la calidad de los cuidados que proporcionan, por ejemplo, deciden la acción de Enfermería apropiada para el momento (1).

Meleis (2) afirma que la Enfermería se enfoca en diferentes aspectos del cuidado, en diferentes momentos de este y con diferentes propósitos; por esta razón, Enfermería no se puede ejemplificar a través de un sólo modelo conceptual o teoría, ya que la disciplina contempla distintas visiones, muchas veces contradictorias. Pero esto no es algo negativo, sino que facilita la inclusión de la diversidad de pensamiento y genera oportunidades de desarrollo tanto para la práctica como para la investigación.

Los profesionales de Enfermería se encargan de proporcionar cuidados de alta calidad y con buena relación costo-efectividad, por lo tanto, la asistencia proporcionada por ellos debe ser evaluada y mejorada constantemente en función de conocimientos de investigación nuevos y mejorados ${ }^{(3)}$. Además, deben tener una mirada crítica en su actuar y preguntarse si sus acciones están sustentadas por el conocimiento científico o son producto de la imitación (4).

Según Fawcett ${ }^{(5)}$ las teorías de rango medio (TRM) tienen como objetivo disminuir la abstracción de las teorías para acercarlas a la práctica y hacer más sencilla su aplicación, dentro de estas teorías se encuentra la teoría del confort de Katherine Kolcaba; La palabra "confort" proviene del inglés comfort, la cual no tiene una traducción específica ${ }^{(6)}$, por lo tanto se mantuvo este término por ser el más utilizado en las publicaciones en español sobre la teoría de Kolcaba, se utiliza durante este artículo como sinónimo de comodidad y bienestar subjetivo. Kolcaba define el Confort como "la experiencia inmediata de sentirse fortalecido al satisfacer las necesidades de alivio, tranquilidad y trascendencia en cuatro contextos (físico, psicoespiritual, sociocultural y ambiental). El confort es mucho más que la ausencia de dolor o de comodidad física" (7). La teoría del confort ha sido ampliamente utilizada en investigaciones ${ }^{(8-14)}$ debido a su simplicidad y claridad en los conceptos, además de ser un aspecto medular de la disciplina de Enfermería, ya que al cuidar se busca el bienestar del paciente, lo que está estrechamente relacionado con el concepto de confort descrito por Kolcaba.

El conocimiento teórico debe guiar la práctica, la educación y la investigación en Enfermería (15), sin embargo, en ocasiones las universidades no le dan la importancia correspondiente, sino que tienden al enfoque biomédico 
y técnico ${ }^{(16)}$. Es por esta razón que muchos profesionales ejercen sin tener presente este conocimiento teórico que fundamenta su quehacer diario, pero aun así realizan actividades que se corresponden con teoristas enfermeras (17).

\section{Por lo expuesto, los objetivos fueron:}

1. Identificar el conocimiento sobre las teorías generales de Enfermería y las TRM, la utilización de teorías en la práctica y ejemplos sobre aplicación de la TRM del confort de Katherine Kolcaba en el quehacer diario de Enfermería en la Unidad de Pabellón, Anestesia y Recuperación del Hospital Clínico Regional Dr. Guillermo Grant Benavente de Concepción, Chile.

2. Aplicar una intervención educativa como una actividad de gestión en la formación continua de los profesionales de Enfermería antes mencionados.

\section{METODOLOGÍA}

Se trató de un proyecto piloto de intervención en gestión educativa, adscrito al enfoque metodológico mixto de carácter exploratorio ${ }^{(18)}$. Se realizó en el contexto de una asignatura del programa de Magíster en Enfermería de la Universidad de Concepción, mediante el convenio docenteasistencial con el Hospital Dr. Guillermo Grant Benavente y con autorización de la enfermera supervisora de la unidad, debido al tiempo, las características del taller y a la voluntariedad en la participación la actividad no se sometió a un comité de ética e investigación. Se realizaron 3 sesiones educativas, entre abril y mayo del 2017, con un muestreo por conveniencia ${ }^{(18)}$ donde asistieron los profesionales de Enfermería que estuvieran disponibles en ese momento $y_{\text {, }}$ mediante la firma de una autorización simple, aceptaron participar voluntariamente, con resguardo de sus datos personales y autorización de publicación de los resultados.

La intervención educativa comenzó con la aplicación de un cuestionario inicial de elaboración propia, el cual consistía en antecedentes personales como edad, sexo, año de egreso de la universidad, tiempo trabajando en la unidad y sistema de turno más tres preguntas de desarrollo con el objetivo de medir el conocimiento previo de los profesionales sobre las teorías de enfermería, cuyas preguntas de desarrollo fueron: "¿Qué son las teorías en Enfermería? ¿Conoce alguna?" "¿Sabe qué son las teorías de rango medio en Enfermería? Si conoce alguna, nombre sus características principales" "¿Aplica alguna teoría de Enfermería en su quehacer diario? ¿Cuál? ¿Cómo?" Finalizado este cuestionario se daba inicio a la presentación audiovisual realizada por la autora principal sobre la "Importancia del conocimiento teórico en Enfermería", donde se explicó el concepto de Enfermería como una disciplina científicohumanista ${ }^{(19)}$, la estructura de este conocimiento según Fawcett, desde lo más abstracto (Metaparadigma) hasta lo más concreto (indicadores empíricos) pasando por las TRM
(5); Enfermería basada en evidencia, la cual implica brindar cuidados basados en investigaciones científicas (3), los principales componentes de la TRM del confort de Kolcaba (1) y ejemplos de su aplicación en la práctica, en donde se destacó el confort físico, psicoespiritual y ambiental (8-10).

Cabe mencionar que los temas expuestos en la educación fueron seleccionados de acuerdo a los objetivos a lograr. Posteriormente se dio paso a un grupo focal denominado "mesa redonda" en donde se invitó a los profesionales a discutir ejemplos de aplicación de la teoría del Confort en la práctica de Enfermería en la unidad, los cuales fueron grabados. Se dio término a la sesión con un cuestionario final, de elaboración propia, con el objetivo de evidenciar comprensión del conocimiento expuesto en la educación, cuyas preguntas de desarrollo fueron: “¿Cuál cree que es la importancia de las teorías en Enfermería?" "De lo conversado en la mesa redonda, exponga sus conclusiones finales sobre la aplicación de la teoría del confort en la unidad".

Es importante señalar que los cuestionarios iniciales y finales no corresponden a un pre y post test para validar la intervención, ya que su objetivo era de recolección de información. A su vez, el control de calidad de los datos se obtuvo a través del levantamiento de esta información con los cuestionarios de manera individual con los participantes y de manera grupal a través de la educación realizada.

Se realizó primeramente el análisis cuantitativo de las variables sociodemográficas mediante el programa estadístico SPSS versión 23.0, posteriormente las grabaciones de la mesa redonda fueron transcritas a Microsoft Word, para finalizar con el análisis de contenido de las respuestas de los cuestionarios y los discursos de los grupos focales en el software Atlas.ti.

\section{RESULTADOS}

Participaron 11 de los 26 enfermeros/as que trabajan en la unidad, los cuales compusieron 2 grupos de 4 personas y 1 grupo de 3 personas. Del total de participantes, 8 fueron mujeres y 3 hombres. La edad promedio fue de 35 años, con un rango de edad de 24 a 61 años. Respecto al tipo de turno hubo 4 enfermeros/as de cuarto turno y 7 de turno diurno. En relación al año de egreso oscila desde 1988 hasta el 2015. Finalmente, el tiempo promedio trabajando en la unidad fueron 6.8 años, con un rango desde 3 meses a 27 años.

Con respecto al cuestionario inicial la primera pregunta buscaba identificar el conocimiento sobre las teorías generales de Enfermería, en donde gran parte de los profesionales de Enfermería definieron a las teorías como la base para su quehacer profesional y como un referente sobre el cual se sustenta su trabajo, siendo la más mencionada la teoría de autocuidado de Dorothea Orem, seguida por la teoría de las 14 necesidades de Virginia Henderson, nombrando también a Callista Roy, Betty Neuman y Hildegard Peplau. La pregunta número dos pretendía determinar el 
conocimiento sobre las TRM en Enfermería, en donde sólo 1 profesional de Enfermería mencionó la teoría del Confort, pero sin características. La pregunta número tres buscaba determinar si utilizaban alguna teoría de Enfermería en un quehacer diario 7 participantes no contestaron la pregunta y 4 mencionaron la teoría del autocuidado, de los cuales sólo 2 describieron actividades que realizaban que se basaban en esa teoría.

Al análisis de los discursos del grupo focal sobre la aplicación de la Teoría del confort en la unidad se agruparon en los 4 contexto del confort descritos por Kolcaba, siendo nombrados principalmente el confortfísico y el psicoespiritual. En el físico destacó la valoración y el manejo del dolor, el cual se manejaba principalmente con la administración de analgésicos endovenosos, además se destacan: cambios de posición, rollos de gel para evitar úlceras por presión, frazadas - mantas térmicas para aumentar la temperatura, entre otros. En el psicoespiritual destacó la comunicación directa con el paciente, especialmente explicando los procesos a los cuales se verá enfrentado, puesto que el someterse a una cirugía es un proceso de estrés para la persona, se ha evidenciado que la información recibida por el paciente, de forma sencilla y comprensible produce una disminución de su ansiedad general, llevando a una recuperación óptima (20). Siguiendo con el confort sociocultural el discurso giró en torno a la familia del paciente, los profesionales de Enfermería relataron que informar a la familia es fundamental en su cuidado, dado que el mismo se encuentra más preocupado por ellos que por su propia condición, a su vez relataron que en el caso del postoperatorio infantil permiten ingresar a un padre del niño, por ejemplo, para consuelo del llanto. Con respecto al confort ambiental se comentó la influencia de factores ambientales que podrían irrumpir en la tranquilidad del paciente provocando una disminución en su confort tales como: intensidad de las luces, ruidos molestos, conversaciones en un tono muy alto, entre otros.

La pregunta uno del cuestionario final pretendía determinar el grado de importancia atribuido a las teorías de Enfermería, en la cual se obtuvo una diversidad de respuestas, pero se destaca que ningún profesional dejó la pregunta en blanco y las respuestas fueron considerablemente más extensas que las del cuestionario inicial. Las respuestas estuvieron englobadas en dos grandes grupos: 1) Las teorías de Enfermería sustentan la práctica profesional y 2) Ayudan a brindar una mejor atención al paciente. Referente a la pregunta dos, la cual pretendía que los profesionales de Enfermería se dieran cuenta que aplicaban la teoría del Confort de Katherine Kolcaba en su quehacer diario en la unidad, al analizar sus respuestas se puede comprobar que se logró ese objetivo ya que, pudieron evidenciar acciones que realizaban diariamente en donde aplicaban la teoría sin conocerla. Gran parte de las respuestas mencionaban los 4 contextos del confort descritos por Kolcaba, y al igual que la pregunta anterior, se englobaban en dos grupos:1) La teoría como sustento de la disciplina de enfermería y 2) Brindar un cuidado de forma integral a los pacientes.

\section{DISCUSIÓN}

Se trabajó con un grupo muy variado de participantes, sin embargo, se obtuvieron respuestas similares en los cuestionarios y acuerdos de opinión en la mesa redonda.

Se pudo determinar el conocimiento previo a la intervención educativa sobre las grandes teorías y las teorías de rango medio en Enfermería, lo cual con respecto a la primera fue escaso y sobre la última fue nulo, lo que nos hace cuestionarnos la importancia otorgada en las mallas curriculares de los profesionales de Enfermería al conocimiento propio de la disciplina. Al finalizar la presentación varios profesionales de Enfermería comentaron que no conocían la existencia de las TRM, que durante su formación no se las habían enseñado, no es de extrañar en los que egresaron de la universidad hace algunos años, pero existían enfermeras egresadas en los años 2014-2015, que las desconocían. Otros comentaron que dentro del hospital no se realizan capacitaciones sobre temas propios de Enfermería, sino sobre procedimientos técnicos y clínicos, con enfoque biomédico, además manifestaron su interés en participar de estos tipos de oportunidades de perfeccionamiento más enfocados en la disciplina de Enfermería.

Se observó que la mayoría de los profesionales de Enfermería no identifica el uso de una teoría o modelo de Enfermería que guíe su quehacer diario, dado que sólo 4 de los 11 profesionales reconocieron utilizar una teoría de Enfermería. Un cuestionamiento importante surge de la incongruencia en las respuestas 1 y 3 del cuestionario inicial; ¿Cómo pueden afirmar que las teorías son la base del quehacer de Enfermería si no son capaces de identificar alguna que practiquen día a día? Sin embargo, luego de realizar la intervención educativa sobre la teoría del confort, los profesionales de Enfermería, durante la sesión de mesa redonda, identificaron fácilmente ejemplos de su aplicación en su trabajo diario. Además, en el cuestionario final se apreció en las respuestas una mejor comprensión del tema y compromiso al responder, evidenciado por una mayor extensión de las mismas y un lenguaje asociado con los conceptos revisados durante la educación. Por lo expuesto anteriormente se podría deducir que los profesionales de Enfermería lograron identificar las teorías de Enfermería como un elemento importante en su práctica profesional luego de la intervención.

Este deficiente conocimiento inicial y falta de uso de las teorías en la práctica clínica de Enfermería, aumenta la brecha entre el saber teórico y el saber práctico definido por Patricia Benner (21), en donde los profesionales de Enfermería dedicados al ámbito asistencial no reconocen las teorías como guías para su práctica diaria, a su vez no realizan investigación debido a que no se generan las instancias necesarias dentro del hospital para este objetivo ${ }^{(22)}$. Por otro lado, se pudo observar que las capacitaciones existentes realizadas a las enfermeras no están enfocadas en lo más importante: el conocimiento propio de Enfermería, sino que se forman en el ámbito biomédico, lo que produce una disminución de la autonomía (23). 
Muchos han sido los esfuerzos de diversos autores para insertar los modelos y teorías de enfermería en la práctica profesional de la misma. El 2014 se realizó una revisión sistemática sobre la implementación de modelos de Enfermería en unidades de paciente crítico, concluyendo que era una tarea difícil la cual necesitaba ciertos puntos clave como: contar con el apoyo institucional, el conocimiento de una docente o enfermera experta y un liderazgo persistente y motivador de manera de transmitirlo al equipo con el que se desea trabajar ${ }^{(24)}$.

A su vez, en el 2008, se realizó un estudio similar al presentado, en donde comenzaban de la premisa que Enfermería depende de la existencia de un conocimiento teórico para todas las áreas. Usar un modelo conceptual - teoría de Enfermería en la atención, garantiza que los profesionales compartan la misma concepción del metaparadigma y lenguaje en la orientación de los cuidados. Los objetivos del estudio fueron conocer el posicionamiento de los profesionales de enfermería de la unidad nefrológica sobre modelos conceptuales y teorías de enfermería y conocer el nivel de aplicabilidad sobre los mismos. En donde observaron una gran variabilidad en cuanto al posicionamiento teórico de los profesionales, ya que se identificaban con distintas teoristas enfermeras ${ }^{(25)}$.

El 2015 se reflexionó sobre el tema de la educación en Enfermería, tanto como proceso de formación universitaria como el de formación continua dirigido a los profesionales, plantea que el desarrollo profesional continuo ha sido reforzado por los avances tecnológicos y por los cambios en el mundo del trabajo, que llevan a las organizaciones y a los individuos a adquirir y actualizar conocimientos (26). Por esto es fundamental las capacitaciones a los profesionales de Enfermería, pero no sólo de los procesos técnicos ni conceptos biomédicos, sino del propio conocimiento científico de Enfermería, educar sobre la búsqueda de investigaciones para realizar un cuidado basado en evidencia y aún más macro, enseñar de forma didáctica y reflexiva los modelos y teorías de enfermería, especialmente las TRM las cuales están pensadas para su aplicación en la práctica, para que las enfermeras asistenciales se identifiquen con una teoría, puedan ver y realizar el cuidado a la persona de una forma propia de Enfermería y no basados en el modelo biomédico.

\section{CONCLUSIONES}

Se espera con este proyecto piloto aportar en la solución de la problemática que se encuentra presente entre los profesionales de Enfermería, evidenciar esta falta de conocimiento y capacitaciones sobre el tema dan luces sobre la necesidad de las mismas. Se debe buscar disminuir la brecha entre el conocimiento teórico y el práctico, el cual constituye un desafío permanente a abordar.

Se recomienda realizar más intervenciones de este tipo, donde se refuercen los conocimientos disciplinares a los profesionales sobre las teorías y prácticas propias de la Enfermería, para nunca olvidar los cimientos de nuestra disciplina, especialmente mostrar la existencia de las TRM en Enfermería cuyo objetivo es disminuir la abstracción de modelos conceptuales y grandes teorías ${ }^{(5)}$, las cuales están pensadas para su aplicación en la práctica es por esto que debemos socializar su existencia entre colegas. A su vez, realizar capacitaciones sobre búsqueda bibliográfica en revistas científicas para identificar intervenciones de Enfermería basadas en la evidencia, especialmente los meta-análisis, y así las enfermeras realicen un cuidado comprobado, efectivo y eficaz (27). Por último, se debe incentivar la formación de grupos de trabajo entre profesionales de Enfermería clínicos y académicos para realizar investigación, ya que los primeros conocen los problemas derivados de la asistencia que afectan directamente a nuestros pacientes y los segundos tiene el conocimiento metodológico para realizar investigación en Enfermería, buscando disminuir la brecha existente entre el conocimiento práctico y el teórico.

\section{CONFLICTOS DE INTERESES}

Las autoras declaran no tener ninguna situación de conflicto de intereses de carácter financiero, académico, político o de otra índole en lo que respecta al desarrollo y publicación de este artículo.

\section{FINANCIAMIENTO}

Las autoras declaran no haber recibido financiamiento para la realización de esta investigación.

\section{REFERENCIAS BIBLIOGRÁFICAS}

1. Alligood MR. Modelos y teorías en enfermería. Barcelona: Elsevier; 2015. 746 p.

2. Meleis Al. Theoretical nursing: development and progress. Fifth Edition. Philadelphia: Wolters Kluwer Health/Lippincott Williams \& Wilkins; 2011.672 p.

3. Burns N, Grove SK. Investigación en enfermería: Desarrollo de la práctica enfermera basada en la evidencia. Barcelona: Elsevier Health Sciences Spain; 2012. 544 p.

4. Tovar-Moncada M, Crespo-Knopfler S. Del aprendizaje vicario al aprendizaje reflexivo en la formación profesional de enfermería. Rev Enferm Inst Mex Seguro Soc. 2015;23(2):115-120.

5. Fawcett J, Desanto-Madeya S. Contemporary nursing knowledge: analysis and evaluation of nursing models and theories. 3rd ed. Philadelphia, PA: F. A. Davis Co; 2013. 453 
p.

6. Real Academia Española de la Lengua. Extranjerismos - préstamos. Ortografía básica de la lengua española, 2010. Disponible en: http://hispanoteca.eu/ gram\%C3\%A1ticas/Gram\%C3\%A1tica\%20espa\%C3\%B1ola/ Ortograf\%C3\%ADa-\%20RAE\%202010-Extranjerismos.htm.

7. Peterson SJ, Bredow TS, editores. Middle range theories: application to nursing research. 3rd ed. Philadelphia: Wolters Kluwer/Lippincott Williams \& Wilkins Health; 2013. 356 p.

8. Townsend CS, Bonham E, Chase L, Dunscomb J, McAlister S. A Comparison of Still Point Induction to Massage Therapy in Reducing Pain and Increasing Comfort in Chronic Pain: Holist Nurs Pract. 2014;28(2):78-84.

9. Seyedfatemi N, Rafii F, Rezaei M, Kolcaba K. Comfort and Hope in the Preanesthesia Stage in Patients Undergoing Surgery. J Perianesth Nurs. junio de 2014;29(3):213-20.

10. Krinsky R, Murillo I, Johnson J. A practical application of Katharine Kolcaba's comfort theory to cardiac patients. Appl Nurs Res. mayo de 2014;27(2):147-50.

11. Egger-Rainer A, Trinka E, Höfler J, Dieplinger AM. Epilepsy monitoring - The patients' views. Epilepsy Behav. marzo de 2017; 68:208-15.

12. Kolcaba K, DiMarco MA. Comfort Theory and its application to pediatric nursing. Pediatr Nurs. junio de 2005;31(3):187-94.

13. Koehn ML. Alternative and complementary therapies for labor and birth: an application of Kolcaba's theory of holistic comfort. Holist Nurs Pract. octubre de 2000;15(1):66-77.

14. March A, McCormack D. Nursing Theory-Directed Healthcare: Modifying Kolcaba's Comfort Theory as an Institution-Wide Approach. Holist Nurs Pract. marzo de 2009;23(2):75-80.

15. Carrillo Algarra AJ, García Serrano L, Cárdenas Orjuela CM, Díaz Sánchez IR, Yabrudy Wilches N. La filosofía de Patricia Benner y la práctica clínica. Enferm Glob. 2013;12(32):346361.

16. Riveros ER, Prieto AM, Flórez-Torres I. Enfermería para el Siglo XXI/Teoría de la Enfermería ¿Un camino de herradura? Aquichan. 2008; 8(1). Disponible en: http://aquichan. unisabana.edu.co/index.php/aquichan/article/view/119

17. Fergusson M, Elisa M. Importancia de los modelos conceptuales y teorías de enfermería: experiencia de la Facultad de Enfermería de la Universidad de La Sabana. Aquichán. octubre de 2005;5(1):44-55.
18. Hernández R, Fernández C, Baptista P, Méndez S. Metodología de la investigación. 6 ed. México D.F. 2014. 600 p.

19. Moreno ME, Muñoz L. De la teoría de enfermería a la práctica, experiencias con proyectos de gestión del cuidado. Universidad de la Sabana: Colección compilaciones, Facultad de Enfermería y Rehabilitación; 2016. 372 p.

20. Marín MD, Cortes AL, Sanz YM, Serrano RM. Aspectos de la información preoperatoria relacionada con la ansiedad del paciente programado para cirugía. Enferm Glob. 2014;14(1):170-191.

21. Molina P, Jara P. El saber práctico en Enfermería. Rev Cuba Enferm. 2010;26(2):111-117.

22. Sancho Cantus D, Prieto Contreras L. Teorías y modelos en la práctica enfermera: ¿Un binomio imposible? Enferm Glob. julio de 2012;11(27):292-8.

23. Orellana A, Sanhueza O. Competencia en investigación en Enfermería. Cienc Enferm. 2011;17(2):9-17.

24. Avilés L, Soto C. Modelos de Enfermería en Unidades de Paciente Crítico: un paso hacia el cuidado avanzado. Enferm Glob. 2014;13(34):323-329.

25. Varez S, López M, Santos S, Abril D. Perspectiva sobre modelos y teorías de enfermería en el ámbito de nefrología. Rev Soc Esp Enferm Nefrológica. 2008;11(3):178-183.

26. Chrizostimo M, Brandão A. La formación profesional del enfermero: 'estado del arte'. Enferm Glob. 2015;14(4):414445 .

27. Orellana A, Paravic T. Enfermería basada en evidencia: barreras y estrategias para su implementación. Cienc Enferm. 2007;13(1):17-24. 